\title{
Electrochemical Sensor for o-Nitrophenol Based on $\beta$-Cyclodextrin Functionalized Graphene Nanosheets
}

\author{
Jinlong Liu, ${ }^{1,2}$ Yihong Chen, $^{1}$ Yujing Guo, ${ }^{1}$ Fengling Yang, ${ }^{1}$ and Fangqin Cheng $^{1}$ \\ ${ }^{1}$ Research Center for Environmental Science and Engineering, State Environmental Protection Key Laboratory of Efficient Utilization \\ Technology of Coal Waste Resources, Institute of Resources and Environment Engineering, Shanxi University, Taiyuan 030006, China \\ ${ }^{2}$ Institute of Chemical Ecology, Shanxi Agricultural University, Taigu 030801, China
}

Correspondence should be addressed to Yujing Guo; guoyj@sxu.edu.cn and Fangqin Cheng; cfangqin@sxu.edu.cn

Received 30 December 2012; Accepted 21 March 2013

Academic Editor: Yongcheng Jin

Copyright (C) 2013 Jinlong Liu et al. This is an open access article distributed under the Creative Commons Attribution License, which permits unrestricted use, distribution, and reproduction in any medium, provided the original work is properly cited.

\begin{abstract}
An electrochemical sensor for the quantification of o-nitrophenol (o-NP) has been developed based on the $\beta$-cyclodextrin functionalized graphene nanosheets modified glassy carbon electrode (CD-GNs/GCE). The results indicated that CD-GNs showed good electrochemical behavior to the redox of o-NP which is attributed to the combination of the excellent properties of graphene and cyclodextrin. The peak currents possess a linear relationship with the concentration of o-NP in the range of 5-400 $\mu \mathrm{M}$. The detection limit of o-NP reached to $0.3 \mu \mathrm{M}$ on the basis of the signal-to-noise characteristics $(\mathrm{S} / \mathrm{N}=3)$. The peak potentials for the reversible redox waves are not affected by other nitrophenol isomers (m, p-NP), illustrating good selectivity. Furthermore, the developed electrochemical sensor exhibited good stability and reproducibility for the detection of o-NP and could be used to determine o-NP in real water sample.
\end{abstract}

\section{Introduction}

Nitrophenols, an important group of environmental pollutants, are widely distributed in the environment due to their extensive use in the manufacturing of insecticides, pesticides, dyes, plastics, and explosives [1]. o-Nitrophenol (o-NP) is one of the nitrophenols isomers which has exerted significant toxic effects on human beings, animals, and plants. Therefore, the development of a new method capable of rapidly and cost-efficiently detecting o-NP in the low concentration is highly desirable and urgently necessary for the environment and health protection. Several instrumental techniques are routinely utilized for the determination of o-NP including high-performance liquid chromatography [2,3], UV-vis, fluorescence spectroscopies $[4,5]$, capillaryzone electrophoresis [6] and electrochemical techniques [7, 8]. Among all these techniques mentioned above, electrochemical methods have received considerable attention for o-NP analysis due to their sensitivity, simplicity, low cost, and easy for on-site determination $[9,10]$.

Graphene, a one-atom thick and two-dimensional closely packed honeycomb lattice, has received numerous investigations from both the experimental and theoretical scientific communities since the experimental observation of single layers by Novoselov et al. in 2004 [11]. Due to its large specific surface area, strong mechanical strength, excellent conductivity, and electrocatalytic activity, graphene is an excellent candidate for electrodes material [12-15]. For instance, Li's group reported the basic electrochemical properties of reduced GN film-modified GCE, which exhibited enhanced electrocatalytic activity for some electroactive species [16]. Lin's group reported a GN-chitosan nanocomposite film, which provided a favorable microenvironment for GOD and promoted its direct electron transfer at the electrode surface [17]. Zhou et al. [18] demonstrated an advanced electrochemical sensing and biosensing platform based on GNs, which showed more favorable electron transfer kinetics and much higher electrocatalytic activity towards $\beta$ nicotinamide adenine dinucleotide (NADH), free bases of DNA, neurotransmitters, and other biological molecules than those of graphite. All the above studies reveal that graphene can play an important role in accelerating electron transfer and may provide a new opportunity for the development of high-performance electrochemical sensors.

Cyclodextrins (CDs) are cyclic oligosaccharides consisting of (a-1,4)-linked a-D-glucopyranose units, which are 
toroidal in shape with a hydrophobic inner cavity and a hydrophilic exterior. CDs have attracted great interest due to their ability to incorporate suitable guest molecules into the hydrophobic cavity [19]. By combining the unique electronic properties of graphene nanosheets with the good water solubility and high supramolecular recognition of $\beta$ $\mathrm{CD}$, the $\beta$-CD-graphene nanocomposite showed significantly improved electrochemical sensing performance compared to unmodified graphene nanosheets [20].

Herein, $\beta$-cyclodextrin functionalized graphene nanosheets modified glassy carbon electrode (CD-GNs/GCE) was fabricated for the determination of o-NP. Due to the unique properties of graphene and CDs, the CD-GNs modified glassy carbon electrode exhibits excellent supramolecular recognition and shows high electrochemical response to oNP compared with that of the bare GCE and GNs/GCE.

In addition, the electrochemical sensor can recognize oNP with its isomer and shows good selectivity. Moreover, this method is simple, cost efficient, sensitive, and also can be used for the determination of $\mathrm{o}-\mathrm{NP}$ in real water sample.

\section{Experiment}

2.1. Materials. Three nitrophenol isomers were obtained from Aladdin Reagent Inc. and were dissolved by ethanol water solution $(1: 2)$. Graphite was purchased from Alfa Aesar. $\beta$-Cyclodextrin ( $\beta$-CD), hydrazine solution (50 wt.\%), and ammonia solution (25-28wt.\%) were obtained from Beijing Chemical Reagent factory (Beijing, China). Other chemicals were of analytical grade and used without further purification. Water used throughout all experiments was purified with the Millipore system. All the experiments were carried out at room temperature.

2.2. Apparatus. AFM image was taken by using a SPI3800N microscope (Seiko Instruments Industry Co., Tokyo, Japan) operating in the tapping mode with standard silicon nitride tips. Typically, the surface was scanned at $1 \mathrm{~Hz}$ with the resolution of 256 lines/image. Electrochemical measurements were performed with a CHI660C electrochemical workstation (ChenHua Instruments Co., Shanghai, China). A conventional three-electrode system was used, including a saturated calomel electrode (SCE) as reference electrode, a platinum wire as counter electrode, and a bare or modified GCE as working electrode.

2.3. Synthesis of CD-Graphene Hybrid Nanosheets and Pure Graphene. Graphene oxide (GO) was synthesized from natural graphite by Hummers' method with a little modification [21]. CD-GNs were synthesized according to our previous work [20]. Briefly, $20.0 \mathrm{~mL}$ of the homogeneous graphene oxide dispersion $(0.5 \mathrm{mg} / \mathrm{mL})$ was mixed with $20.0 \mathrm{~mL}$ of $\beta$-CD aqueous solution $(80 \mathrm{mg}$ ) and $300.0 \mu \mathrm{L}$ of ammonia solution, followed by the addition of $20 \mu \mathrm{L}$ of hydrazine solution. After being vigorously shaken or stirred for a few minutes, the vial was put in a water bath $\left(60^{\circ} \mathrm{C}\right)$ for $3.5 \mathrm{~h}$. The stable black dispersion was obtained. The dispersion was filtered with a nylon membrane $(0.22 \mu \mathrm{m})$ to obtain CD-GNs that can be redispersed readily in water by ultrasonication.

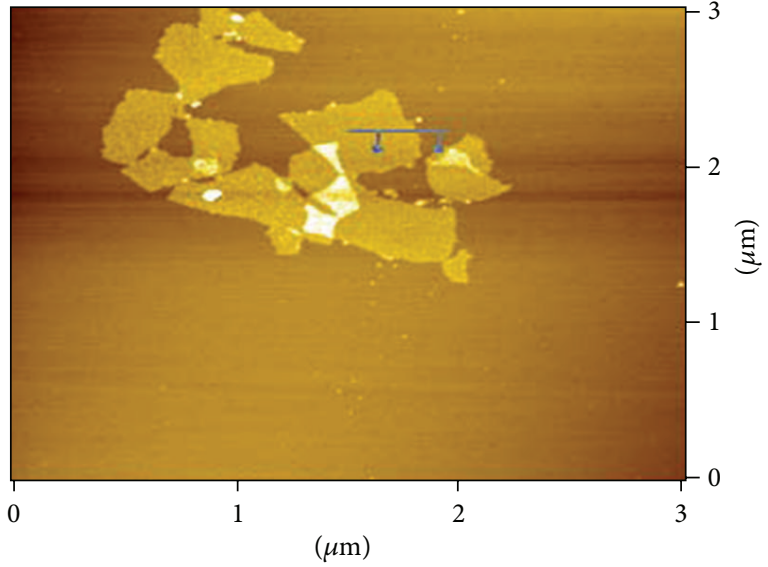

(a)

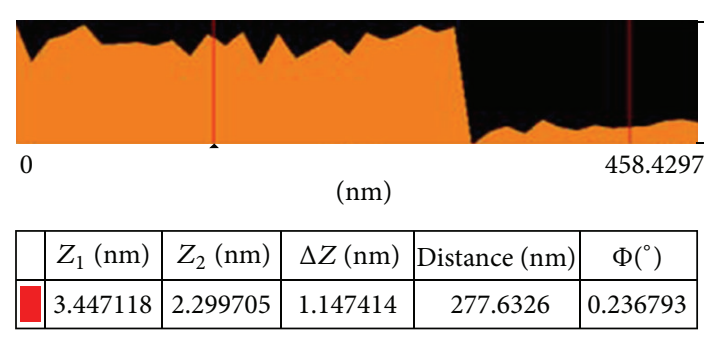

(b)

FIGURE 1: (a) AFM image of CD-GNs. (b) The cross section identified by the line in Figure 1 shows the height of CD-GNs.

Additionally, the preparation of pure graphene was similar to CD-GNs except for the addition of CD.

\subsection{Preparation of the CD-GNs/GCE or GNs/GCE and the} Electrochemical Measurement. Prior to the modification, the glassy carbon electrode was polished with $1,0.3$, and $0.05 \mu \mathrm{m}$ alumina slurry and rinsed thoroughly with doubly distilled water between each polishing step. Then, it was washed successively with 1:1 nitric acid, acetone, and doubly distilled water in an ultrasonic bath and dried in air. Lastly, $5 \mu \mathrm{L}$ of $0.25 \mathrm{mg} / \mathrm{mL}$ CD-GNs or GNs was carefully cast on the surface of the well-polished GCE and dried in air. The CD-GNs/GCE or GNs/GCE electrode was thus obtained. The electrode was then transferred into $0.1 \mathrm{M} \mathrm{pH} 7.0$ phosphate buffer solution containing o-NP, and the CV or DPV signal was recorded after accumulation for $10 \mathrm{~min}$.

\section{Results and Discussion}

3.1. The Morphology of the CD-GNs. Atomic force microscopy (AFM) can directly characterize the morphologies and layers of CD-GNs. The AFM samples were prepared by dropping CD-GNs dispersed in water onto clean mica surfaces and dried at room temperature. Figure 1 shows the AFM image of CD-GNs, which illustrates the flakelike shapes of graphene. The average thickness of the graphene nanosheets is about 1$2 \mathrm{~nm}$, a typical characteristic of functional molecule protected single-layer graphene [22]. 


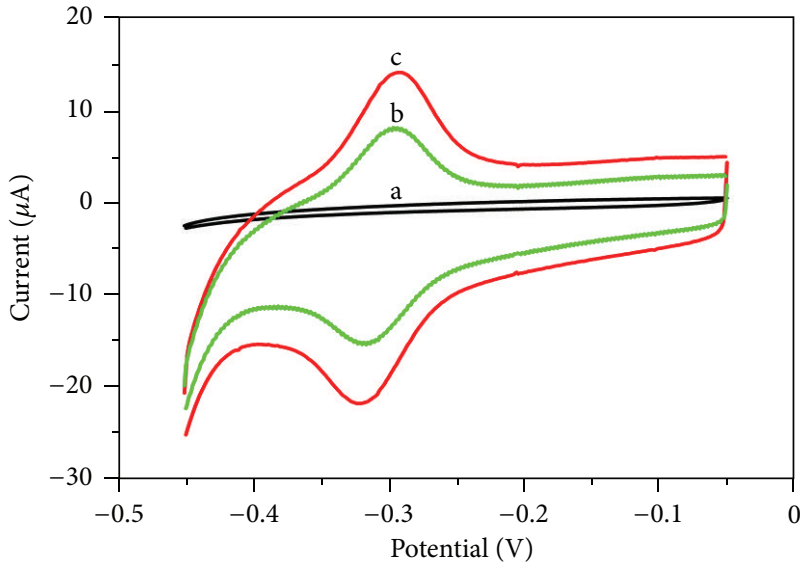

Figure 2: Cyclic voltammograms of $50 \mu \mathrm{M}$ o-NP at GCE (curve a), GNs/GCE (curve b), and CD-GNs/GCE (curve c) in $0.1 \mathrm{M}$ phosphate buffer ( $\mathrm{pH}$ 7.0). Scan rate: $50 \mathrm{mV} \mathrm{s}^{-1}$.

3.2. The Voltammetric Behavior of o-NP. Figure 2 compares the voltammetric behaviors of $50 \mu \mathrm{M}$ o-NP at the bare GCE (a), the GNs/GCE (b), and the CD-GNs/GCE (c) in $0.1 \mathrm{M}$ pH 7.0 phosphate buffer solutions. There are no significant redox peaks at the bare GCE. However, at the GNs/GCE, oNP shows better electrochemical behavior than the bare GCE, which may be attributed to the excellent conductivity and large surface area of graphene. While at the CD-GNs/GCE, the peak currents show a remarkable increase compared to that at the other two electrodes. This demonstrates that $\beta$-CD molecules on the surface of graphene with high supramolecular recognition capability can form host-guest complexes with o-NP. Accordingly, the concentration of o-NP on the electrode has been increased which results in remarkable peak currents enhancement. These phenomena prove that the CD-GNs not only display the excellent properties of graphene, but also exhibit the supramolecular recognition capability of CDs.

The influence of scan rate on the peak current of o-NP at the CD-GNs modified electrode was also investigated. As shown in Figure 3(a), the peak currents of o-NP increased with the increase of the scan rate. At low scan rate (50$\left.300 \mathrm{mVs}^{-1}\right)$, the peak current $\left(i_{\mathrm{p}}\right)$ and the square root of scan rate $(v)$ exhibited well linear relation. The linear regression equations are $i_{\mathrm{pa}}(\mathrm{A})=1.806 \times 10^{-5}-3.06 \times 10^{-6} v^{1 / 2}(\mathrm{~V} / \mathrm{s})^{1 / 2}$ $(r=0.994)$ and $i_{\mathrm{pc}}(\mathrm{A})=-1.13 \times 10^{-5}+1.85 \times 10^{-6} v^{1 / 2}(\mathrm{~V} / \mathrm{s})^{1 / 2}$ $(r=0.998)$ (Figure 3(b)). The ratio of the anodic peak current to cathodic peak current is almost equal to unity $\left(i_{\mathrm{pa}}: i_{\mathrm{pc}}=\right.$ $1)$. These results demonstrate the electrochemistry of the CDGNs modified GCE corresponding to a diffusion-controlled process which is reversible.

3.3. Optimization of the Experimental Conditions. The influence of different conditions such as $\mathrm{pH}$, the amount of CDGNs, and accumulation time on the electroactivity of CDGNs towards o-NP was investigated. As shown in Figures 4(a) and 4(b), the redox peak currents increased by varying $\mathrm{pH}$ from 4.0 to 7.0 and then decreased rapidly when the $\mathrm{pH}$ exceeded 7.0. This phenomenon may be ascribed to the

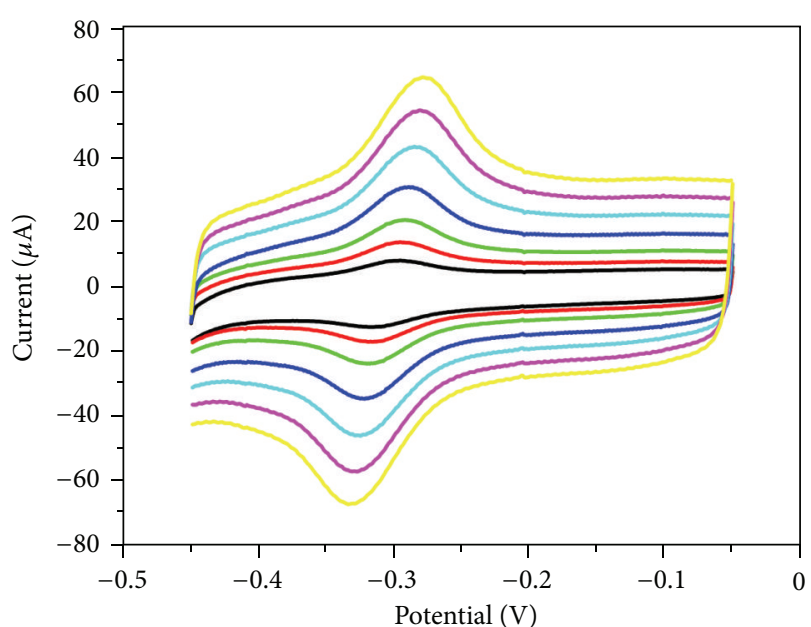

(a)

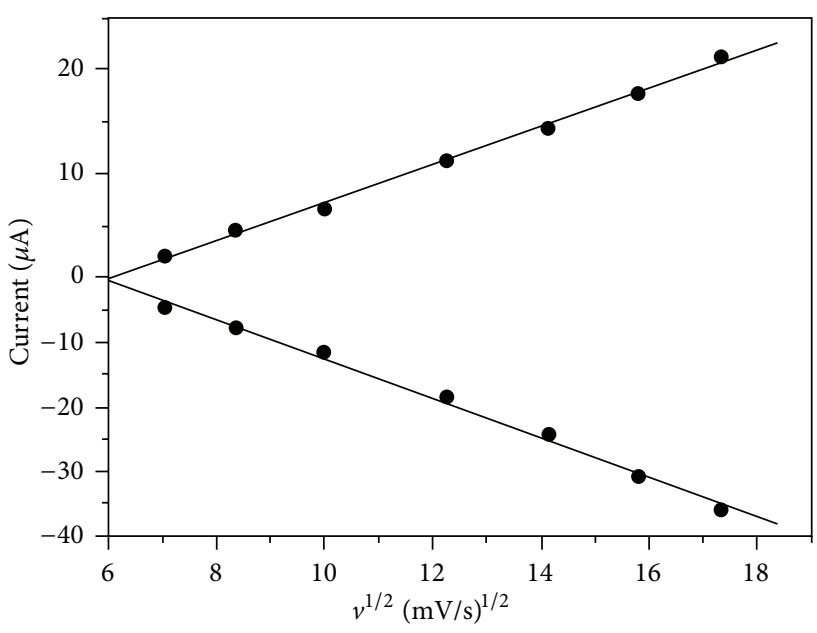

(b)

Figure 3: (a) Cyclic voltammograms of $50 \mu \mathrm{M}$ o-NP in $0.1 \mathrm{M}$ phosphate buffer at the CD-GNs/GCE with different scan rates $(50,70$, $100,150,200,250$, and $300 \mathrm{mV} / \mathrm{s}$ ). (b) The peak currents of o-NP versus the square root of scan rate.

following two reasons: one is that the proton is critical in redox behavior of o-NP, and the decrease of o-NP response at high $\mathrm{pH}$ is possibly due to the decreases of proton concentration. The other is that the change of microenvironment weakened the inclusive ability of $\beta$-CD with o-NP and thereby decreased the o-NP concentration on the electrode and reduced the peak current. Therefore, phosphate buffer with $\mathrm{pH} 7.0$ was selected for the subsequent experiments.

The effect of the amount of CD-GNs is shown in Figure $4(\mathrm{c})$. When the amount of CD-GNs suspension increased from 2 to $5 \mu \mathrm{L}$, the peak currents of o-NP increased dramatically. However, when it exceeded $5 \mu \mathrm{L}$, the peak currents decreased. This may be attributed to the thicker film of CD-GNs, which blocked the electrical conductivity. So, the amount of CD-GNs on the GCE was optimized at $5 \mu \mathrm{L}$.

Figure 4(d) illustrates the effects of accumulation time on the peak currents of o-NP. The peak current increased 


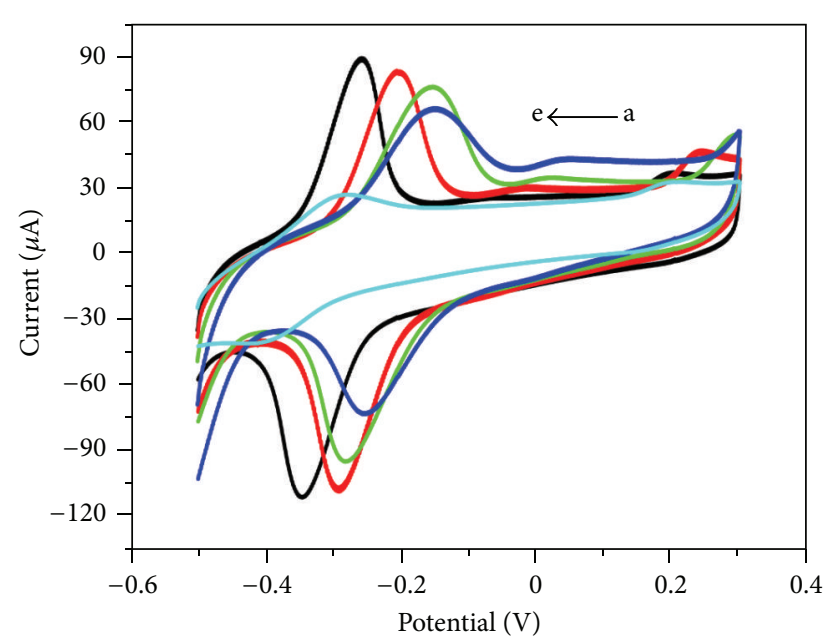

(a)

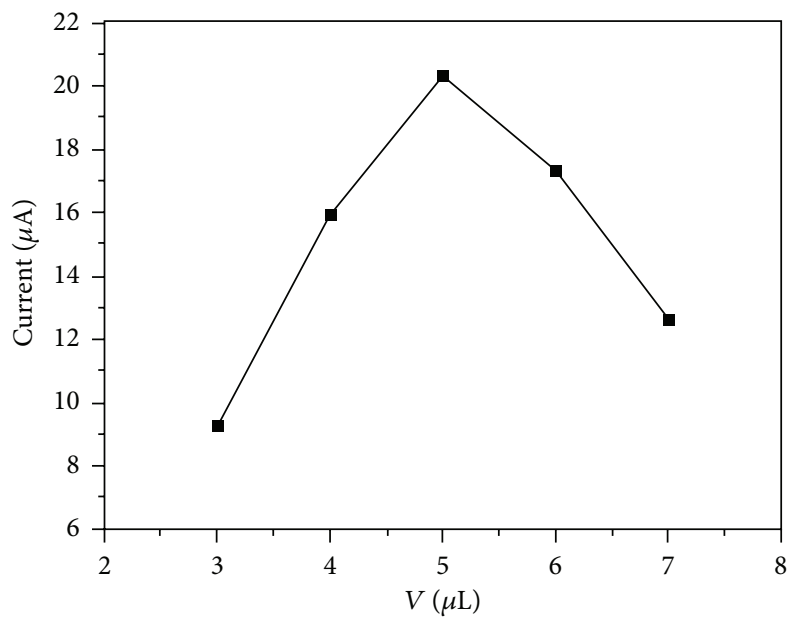

(c)

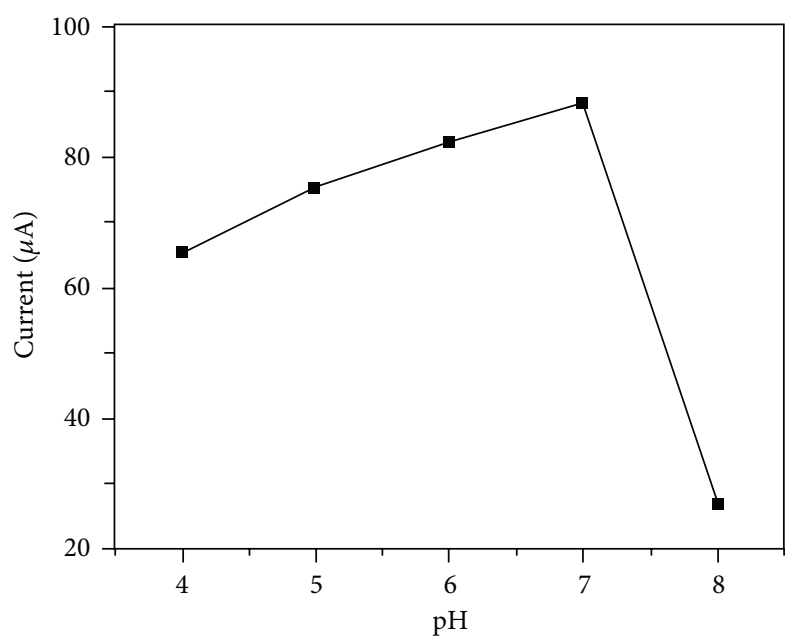

(b)

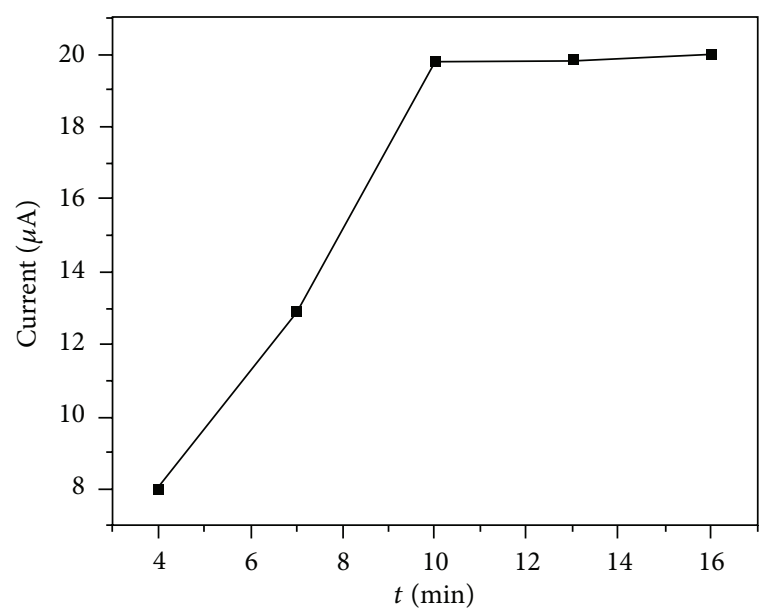

(d)

Figure 4: (a) Cyclic voltammograms of $50 \mu \mathrm{M}$ o-NP at CD-GNs/GCE in a series of phosphate buffer solutions with different $\mathrm{pH}$ (a-e): 4, 5, 6, 7, and 8; (b) the corresponding $\mathrm{pH}$-current plot. (c) The influence of the amount of CD-GNs on the peak currents. (d) The influence of the accumulation time on the peak currents.

with the accumulation time within $10 \mathrm{~min}$, owing to the increased amount of o-NP on the CD-GNs/GCE. Further increment of the accumulation time did not increase the peak current obviously, which indicated the surface adsorption saturation. For practical purposes, $10 \mathrm{~min}$ was sufficient for the determination of o-NP.

3.4. Electrochemical Detection of o-NP. Differential pulse voltammetric (DPV) was used for the determination of o-NP under the optimum conditions. The enhanced peak current was linear with the concentration of o-NP in the range of 5.0-400 $\mu \mathrm{M}$ (Figure 5). The linear equation is $i_{\mathrm{p}}(\mu \mathrm{A})=$ $0.1670+0.4316 \mathrm{c}(\mu \mathrm{M})(R=0.9997)$. Based on the signalto-noise ratio of 3 , the experimental detection limit was obtained as $0.3 \mu \mathrm{M}$. The results are superior or comparable to the method reported previously $[9,23]$. As a novel sensor material for the determination of o-NP, the interference of the other two nitrophenol isomers m-nitrophenol (m-NP) and p-nitrophenol (p-NP) was investigated. In $\mathrm{pH} 7.0$ phosphate buffer solution, cyclic voltammogram of the mixtures containing $0.5 \mathrm{mM}$ o-NP, $\mathrm{m}-\mathrm{NP}$, and $\mathrm{p}-\mathrm{NP}$, respectively, is shown in Figure 6. It is clearly to see that peak potentials for the reversible redox waves of o-NP are not affected by $\mathrm{m}, \mathrm{p}-\mathrm{NP}$ isomers. Therefore, the CD-GNs present good selectivity in the detection of o-NP.

3.5. Real Sample Analysis. In order to evaluate the practical application of the CD-GNs modified GCE, it was used to detect o-NP in several lake water samples. No signals for o-NP were observed in these lake water samples. Thus, the proposed method was applied to the lake water samples spiked with o-NP by adding known amount of o-NP solution. In $\mathrm{pH} 7.0$ phosphate buffer solution, the samples were measured under the same condition for four times. The average recovery and relative standard deviation (RSD) were determined and shown in Table 1 . The recovery values ranged from 97.4 to $102.3 \%$, which indicates that this sensor could be used in the detection of o-NP in real water samples. 


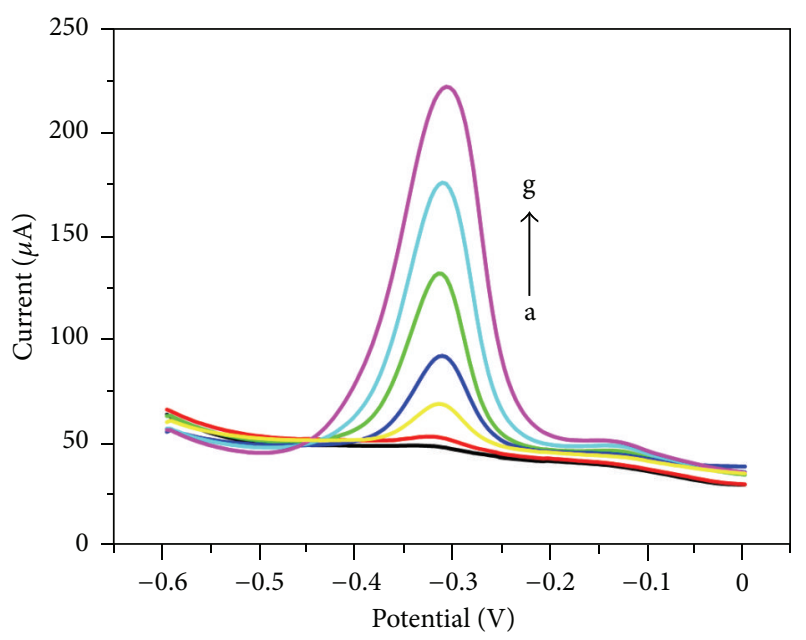

(a)

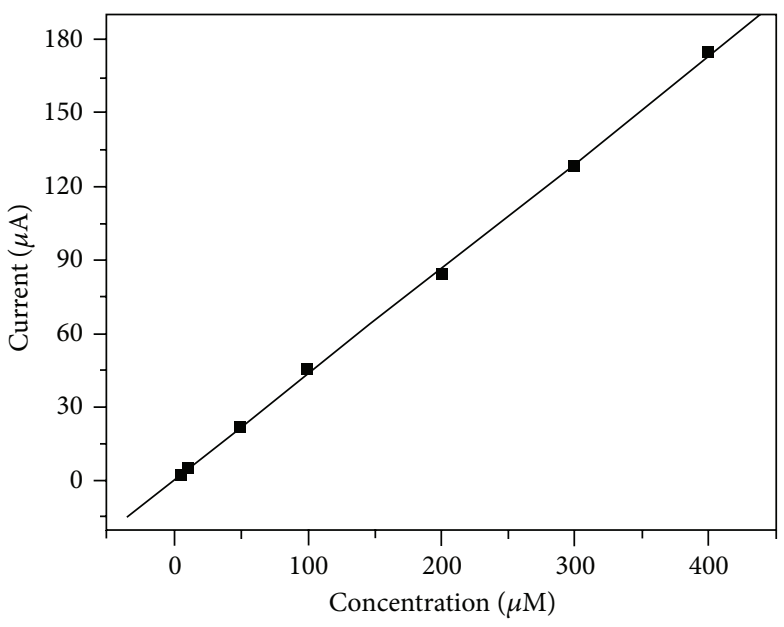

(b)

Figure 5: (a) The DPV response for the different concentrations of o-NP (a-g: 5, 10, 50, 100, 200, 300, and 400 $\mu \mathrm{M}$ ). (b) The calibration plot of o-NP.

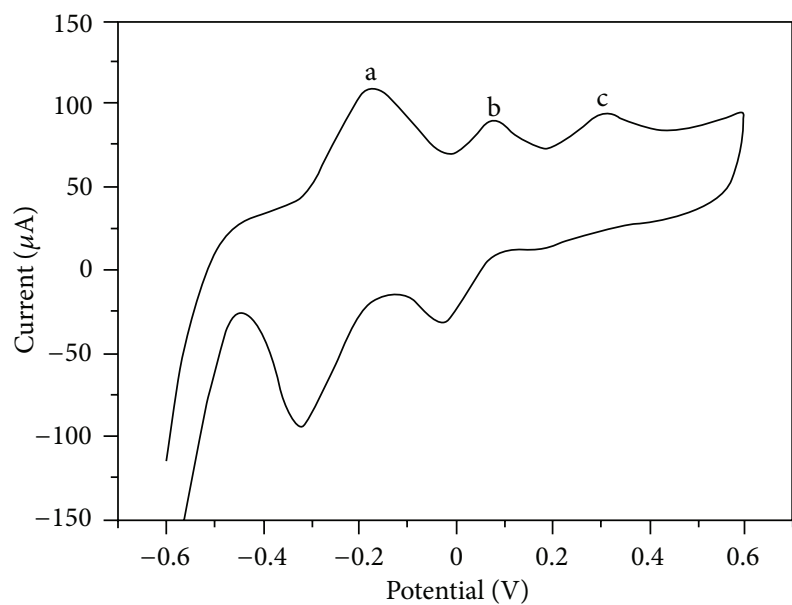

FIGURE 6: The cyclic voltammogram of three nitrophenol isomers. (a) o-NP; (b) m-NP; and (c) p-NP.

3.6. Repeatability and Stability. To estimate the repeatability of the proposed method, the current response of $50 \mu \mathrm{M}$ oNP was detected with the same bare GCE modified six times in the same procedure. The relative standard deviation was calculated to be $4.4 \%$. The results revealed that the electrode possessed satisfying reproducibility. Moreover, the CD-GNsbased o-NP electrochemical sensor also exhibits a good longterm stability. The peak current can maintain over $94 \%$ of its initial value after the modified electrode stored at room temperature for 10 days.

\section{Conclusion}

In this work, CD-GNs were used as enhanced material for the electrochemical determination of o-NP. The simply fabricated CD-GNs-based electrochemical sensor showed superior electrochemical performance for the determination of oNP relative to those of GNs and bare GCE. Moreover, the
TABLE 1: The recoveries of o-NP from water sample.

\begin{tabular}{lcccc}
\hline Sample & Added $(\mu \mathrm{M})$ & Found $(\mu \mathrm{M})$ & Recovery $(\%)$ & RSD $(\%)$ \\
\hline 1 & 10 & 10.12 & 101.2 & 2.6 \\
2 & 20 & 19.48 & 97.4 & 3.2 \\
3 & 50 & 49.93 & 99.9 & 4.4 \\
4 & 80 & 81.84 & 102.3 & 2.5 \\
5 & 100 & 98.61 & 98.6 & 3.5 \\
\hline
\end{tabular}

electrochemical sensor exhibited good selectivity, repeatability, and stability. Thereby, the present CD-GNs-based oNP sensors will probably be promising for a wide range of applications related to the detection of trace amounts of phenol compounds.

\section{Acknowledgments}

This research work was supported by the National Natural Science Foundation of China (no. 21275093) the Natural Science Foundation of Shanxi Province (no. 2012011007-3), the International Scientific and Technological Cooperation of China (2011DFA90830), and the National Hi-Tech Research and Development Program of China (863 Program, 2011AA06A103).

\section{References}

[1] D. R. Hartter, "The use and importance of nitroaromatic chemicals in the chemical industry," in Toxicity of Nitroaromatic Compounds, D. E. Rickert, Ed., pp. 1-14, Chemical Industry Institute of Toxicology, Washington, DC, USA, 1985.

[2] M. J. Thompson, L. N. Ballinger, S. E. Cross, and M. S. Roberts, "High-performance liquid chromatographic determination of phenol, 4-nitrophenol, $\beta$-naphthol and a number of their glucuronide and sulphate conjugates in organ perfusate," Journal of Chromatography B, vol. 677, no. 1, pp. 117-122, 1996. 
[3] T. Galeano-Diaz, A. Guiberteau-Cabanillas, N. Mora-Diez, P. Parrilla-Vazquez, and F. Salinas-Lopez, "Rapid and sensitive determination of 4-nitrophenol, 3-methyl-4-nitrophenol, 4,6dinitro-o-cresol, parathion-methyl, fenitrothion, and parathion-ethyl by liquid chromatography with electrochemical detection," Journal of Agricultural and Food Chemistry, vol. 48, no. 10, pp. 4508-4513, 2000.

[4] A. Niazi and A. Yazdanipour, "Spectrophotometric simultaneous determination of nitrophenol isomers by orthogonal signal correction and partial least squares," Journal of Hazardous Materials, vol. 146, no. 1-2, pp. 421-427, 2007.

[5] C. Nistor, A. Oubi, M. P. Marco, D. Barceló, and J. Emnéus, "Competitive flow immunoassay with fluorescence detection for determination of 4-nitrophenol," Analytica Chimica Acta, vol. 426, no. 2, pp. 185-195, 2001.

[6] X. Guo, Z. Wang, and S. Zhou, "The separation and determination of nitrophenol isomers by high-performance capillary zone electrophoresis," Talanta, vol. 64, no. 1, pp. 135-139, 2004.

[7] Z. Liu, J. Du, C. Qiu et al., "Electrochemical sensor for detection of p-nitrophenol based on nanoporous gold," Electrochemistry Communications, vol. 11, no. 7, pp. 1365-1368, 2009.

[8] A. K. M. Kafi and A. Chen, "A novel amperometric biosensor for the detection of nitrophenol," Talanta, vol. 79, no. 1, pp. 97-102, 2009.

[9] L. Q. Luo, X. L. Zou, Y. P. Ding, and Q. S. Wu, "Derivative voltammetric direct simultaneous determination of nitrophenol isomers at a carbon nanotube modified electrode," Sensors and Actuators B, vol. 135, no. 1, pp. 61-65, 2008.

[10] C. Xu, J. Wang, L. Wan, J. Lin, and X. Wang, "Microwaveassisted covalent modification of graphene nanosheets with hydroxypropyl- $\beta$-cyclodextrin and its electrochemical detection of phenolic organic pollutants," Journal of Materials Chemistry, vol. 21, no. 28, pp. 10463-10471, 2011.

[11] K. S. Novoselov, A. K. Geim, S. V. Morozov et al., "Electric field in atomically thin carbon films," Science, vol. 306, no. 5696, pp. 666-669, 2004.

[12] A. K. Geim and K. S. Novoselov, "The rise of graphene," Nature Materials, vol. 6, no. 3, pp. 183-191, 2007.

[13] H. A. Becerril, J. Mao, Z. Liu, R. M. Stoltenberg, Z. Bao, and Y. Chen, "Evaluation of solution-processed reduced graphene oxide films as transparent conductors," ACS Nano, vol. 2, no. 3 , pp. 463-470, 2008.

[14] M. J. Allen, V. C. Tung, L. Gomez et al., "Soft transfer printing of chemically converted graphene," Advanced Materials, vol. 21, no. 20, pp. 2098-2102, 2009.

[15] D. Li, M. B. Muller, S. Gilje, R. B. Kaner, and G. G. Wallace, "Processable aqueous dispersions of graphene nanosheets," Nature Nanotechnology, vol. 3, no. 2, pp. 101-105, 2008.

[16] L. H. Tang, Y. Wang, Y. M. Li, H. B. Feng, J. Lu, and J. H. $\mathrm{Li}$, "Preparation, structure, and electrochemical properties of reduced graphene sheet films," Advanced Functional Materials, vol. 19, no. 17, pp. 2782-2789, 2009.

[17] X. Kang, J. Wang, H. Wu, I. A. Aksay, J. Liu, and Y. Lin, "Glucose oxidase-graphene-chitosan modified electrode for direct electrochemistry and glucose sensing," Biosensors and Bioelectronics, vol. 25, no. 4, pp. 901-905, 2009.

[18] M. Zhou, Y. M. Zhai, and S. J. Dong, "Electrochemical sensing and biosensing platform based on chemically reduced graphene oxide," Analytical Chemistry, vol. 81, no. 14, pp. 5603-5613, 2009.

[19] M. V. Rekharsky and Y. Inoue, "Complexation thermodynamics of cyclodextrins," Chemical Reviews, vol. 98, no. 5, pp. 1875-1917, 1998.
[20] Y. Guo, S. Guo, J. Ren, Y. Zhai, S. Dong, and E. Wang, “Cyclodextrin functionalized graphene nanosheets with high supramolecular recognition capability: synthesis and host-guest inclusion for enhanced electrochemical performance," ACS Nano, vol. 4, no. 7, pp. 4001-4010, 2010.

[21] W. S. Hummers and R. E. Offeman, "Preparation of graphitic oxide," Journal of the American Chemical Society, vol. 80, no. 6, article 1339, 1958.

[22] C. N. R. Rao, A. K. Sood, K. S. Subrahmanyam, and A. Govindaraj, "Graphene: the new two-dimensional nanomaterial," Angewandte Chemie - International Edition, vol. 48, no. 42, pp. 7752-7777, 2009.

[23] I. N. Rodriguez, M. B. Zamora, J. M. B. Salvador, J. A. M. Leyva, M. P. Hernandez-Artiga, and J. L. H. H. de Cisneros, "Voltammetric determination of 2-nitrophenol at a bentonite-modified carbon paste electrode," Mikrochimica Acta, vol. 126, no. 1-2, pp. 87-92, 1997. 

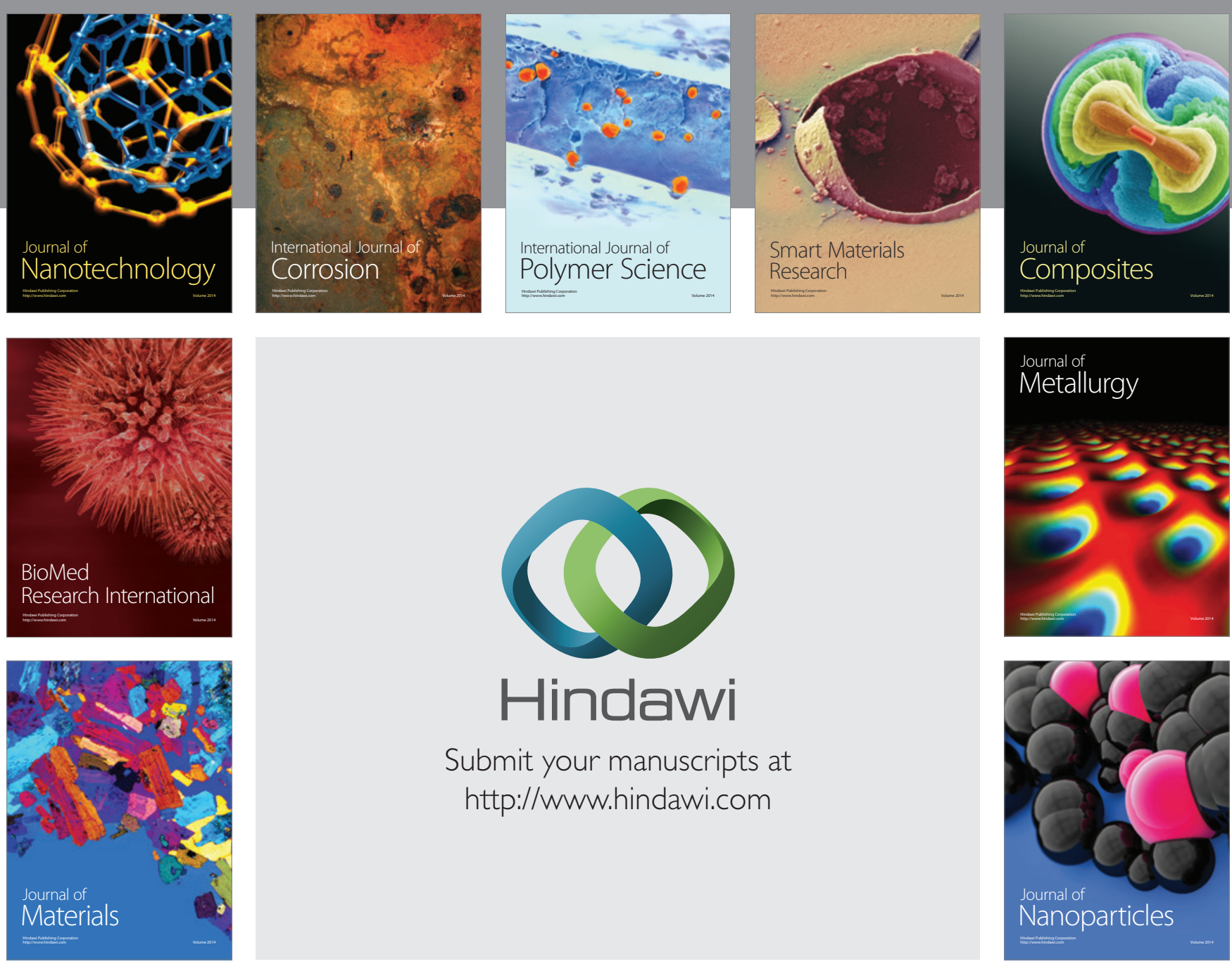

Submit your manuscripts at http://www.hindawi.com
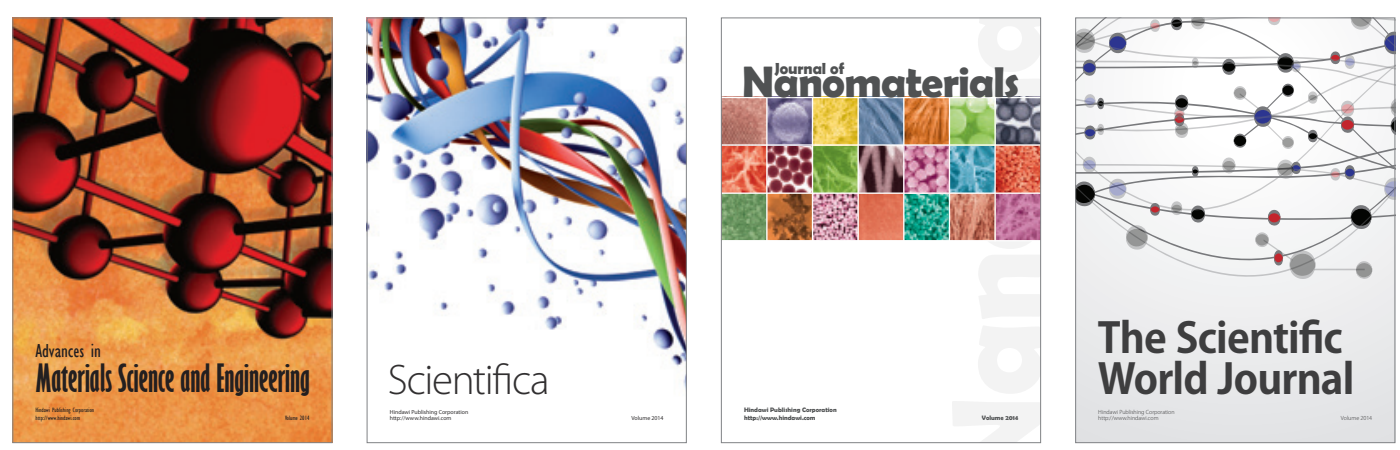

\section{The Scientific World Journal}
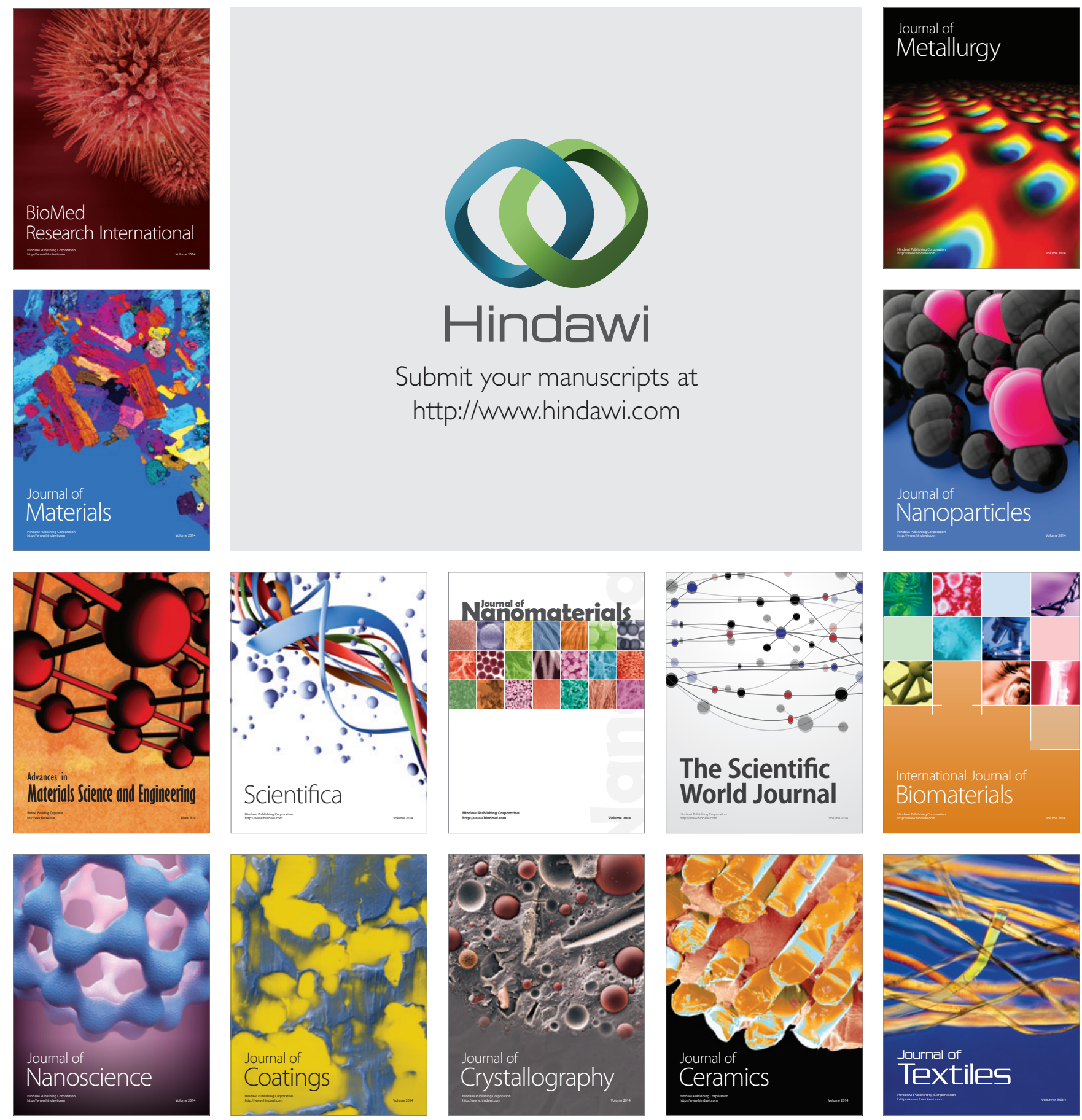